\title{
Recent Advances in Pathologic Research and Targeted Therapies of Thymoma
}

\author{
Lvcheng Jin ${ }^{1, \#}$ and Yun-Xiang Zhang ${ }^{2, \#, *}$ \\ ${ }^{1}$ Department of Pathology, Weifang Medical University, Weifang, Shandong, 261041, China \\ ${ }^{2}$ Department of Pathology, Weifang People's Hospital, Weifang, Shandong, 261041, China
}

\begin{abstract}
Thymoma is a rare tumor that was reclassified by the World Health Organization in 2015. Recent studies have made advances in molecular targeted therapies, such as c-KIT, EGFR, IGF-1R, PTEN, HDAC, VEGF and PD-L1. Additionally, new molecular markers such as CTV/CTS, GTF2I, Pax8 and DSG-3 have been used in the differential diagnosis of thymoma. This article reviews molecular pathogenesis of thymoma, application of molecular pathology in the differential diagnosis of thymoma and recent progress in targeted therapies for thymoma.
\end{abstract}

Keywords: Thymic epithelial tumors, gene therapy, targeted therapy, molecular pathology.

\section{INTRODUCTION}

Thymoma is considered as a thymic epithelial tumor and usually located in anterior superior mediastinum. Thymomas show low-grade malignant potential with local infiltrating growth or rarely distant metastasis [1]. Thymic epithelial tumors have various forms. There have been many changes in the classification of these tumors with controversies. In 2015, the WHO classified thymic epithelial tumors into type $A$, atypical type $A$, type $A B$, type $B 1$, type $B 2$, type $B 3$, and thymic carcinoma [2]. In recent years, a variety of tumor molecular markers have been discovered to aid in the pathologic diagnosis and provide information for molecular targeted therapies.

\section{MOLECULAR PATHOGENESIS OF THYMIC EPITHELIAL TUMORS}

The formation of cancer is a multi-step process involving oncogene activation, tumor suppressor gene inactivation and other changes. Saito $M$ et al. [3] analyzed genomic and epigenetic aberrations in Asian patients with thymic carcinoma using whole exome sequencing, RNA sequencing and methylation array, and then compared the findings with those from American/European patients with thymic carcinoma. They found that molecular process leading to thymic carcinoma depends on accumulation of genetic and epigenetic aberrations.

PTEN (PHOSPHATE and TENSION homology) is located on10q23.3.It has nine exons and encodes a protein phosphatase of 403 amino acids, also called as

\footnotetext{
*Address correspondence to this author at the Department of Pathology, Weifang People's Hospital, Weifang, Shandong, 261041, China;

E-mail: zhangbing199592@163.com

${ }^{\#}$ The authors contributed equally to this work.
}

mutated in multiple advanced cancer 1 (MMAC1) or TGF-regulated and epithelial cell -enriched phosphatase (TEP1). Some studies have found inactivation of PTEN and over expression of c-myc occurred in thymoma and their expressions were negatively correlated. These two genes seem to play a synergistic role in malignant transformation of thymic epithelial cells, leading to development of thymoma [4]. Masunaga $\mathrm{A}$ et al. [5] analyzed genetic mutations and RNA expression in two non-tumor thymus specimens, 33 thymomas and 4 thymic carcinomas using direct sequencing, methylation-specific PCR and reverse transcription PCR (RT-PCR). The results demonstrated that PTEN protein was not detected in non-tumor thymus tissues or B1/B2 thymoma tissues, but was detected in type A thymoma and thymic carcinoma tissues. The findings suggest that non-tumor thymic epithelial cells and B1/B2 thymoma cells have a mechanism of translational inhibition and/or accelerated protein degradation, whereas type A thymoma cells exhibit transcriptional repression of mRNA and accelerate translation and/or protein accumulation.

Wang et al. [6] studied Wnt4 and Forkhead box protein N1 (FoxN1) in thymoma and found that the expressions of Wnt4 and FoxN1 were positively correlated, suggesting their involvement in thymoma pathogenesis.

\section{TARGETED THERAPIES FOR THYMIC EPITHELIAL TUMORS}

Due to their low incidence and unclear pathogenesis, there are no effective molecular targeted therapies for thymic epithelial tumors, comparing to non-small cell lung carcinoma. Recurrence or metastasis of thymic epithelial tumors may still occur 
after surgical resection and chemotherapy and/or radiotherapy [7]. However, studies of specific molecular markers have promoted the exploration of new molecular targeted therapies in thymic epithelial tumors.

\subsection{Programmed Death-Ligand-1}

Expression of programmed death-ligand-1 (PD-L1) has been detected in various tumors including thymic epithelial tumors. Katsuya $\mathrm{Y}$ et al. [8] found PD-L1 was expressed in thymoma and thymic carcinoma using tissue microarray, suggesting potentials of anti-PD1/PD-L1 immunotherapy in unresectable or recurrent thymoma/thymic carcinoma. Enkner $\mathrm{F}$ et al. [9] analyzed the expression of multiple proteins including anaplastic lymphoma kinase (ALK), human epidermal growth factor receptor 2 (HER2), human epidermal growth factor receptor 3 (HER3), MET, phosphorylation of mammalian rapamycin Target protein (mTOR), P16, platelet-derived growth factor receptor alpha (PDGFRA), human beta-type platelet-derived growth factor receptor (PGFGFB), PD-L1, PTEN and ROS1 in 37 thymomas and 35 thymic carcinomas. The results demonstrated that PDGFRA was highly expressed in thymic carcinoma and PD-L1 was expressed in B3 thymoma and thymic carcinoma, suggesting potential therapeutic targets in these tumors.

Tiseo $M$ et al. [10] analyzed the expression of EGFR, C-KIT, kirsten rat sarcoma viral oncogene (KRAS), ALK and PD-L1 by immunohistochemistry (IHC) in 112 thymoma cases and correlated with clinicopathological features. The results demonstrated that thymic carcinoma may carry C-KIT mutations and increased PD-L1 expression. Our group examined PD$\mathrm{L} 1$ protein and mRNA in tumor cells and stromal tumor infiltrating lymphocytes using $\mathrm{IHC}$ and real-time fluorescent quantitative PCR in 70 thymic epithelial tumors. The results demonstrated that PD-L1 protein positive rate (cut-off value: $1 \%$ ) was $76.7 \%$ in $\mathrm{B} 3$ thymoma or thymic carcinoma, significantly higher than that in other types (37.5\%). PD-L1 protein expression was significantly associated with histological type, Masaoka staging, radiotherapy and chemotherapy $(P<0.05)$. PD-L1 mRNA expression was positively correlated with PD-L1 protein expression and the overall coincidence rate of the two methods was $85.71 \%$. Our and others' findings suggest that PDL1/PD-1 blockage may be a new strategy for immunotherapy in thymic epithelial tumors, especially in B3 thymoma or thymic carcinoma [11].

\subsection{C-Kit (CD117)}

C-Kit, a retroviral proto-oncogene encoding a transmembrane receptor with tyrosine kinase activity, was firstlydetected in healthy cats in 1986 [12]. C-Kit has been found to be overexpressed in thymic carcinoma, but rarely in thymoma [13-15]. Schirosi $L$ et al. [16] studied the protein expression of c-Kit, p63, CD5 and neuroendocrine markers using IHCs, and genetic mutation of c-Kit exon 9, 11, 13, 14 and 17 in 48 thymic carcinomas using direct sequencing. They found that c-Kit was positive in $60 \%$ of cases, CD5 positive in $69 \%$, and p63 in $85 \%$. Six c-Kit protein positive cases $(12.5 \%)$ showed c-Kit gene mutations. No genetic mutations were detected in c-Kit protein negative tumors. C-Kit genetic mutations were found in all poorly differentiated thymic squamous cell carcinomas expressing c-Kit protein, CD5 and p63, but lacking neuroendocrine markers. The mutations included mutations in exon 11 (V559A, L576P, Y553N, W557R), in exon 9 (E490K) and in exon 17 (D820E). Since c-Kit mutation can predict the efficacy of c-Kit inhibitors, the results suggest all thymic carcinomas should be screened using IHCs for c-Kit and then c-Kit genetic mutation analysis may be performed only in cKit protein positive cases, especially with concurrent positive CD5 and p63, but negative neuroendocrine markers.

Imatinib, a small molecule tyrosine kinase inhibitor, has the effect of blocking one or more protein kinases. It is commonly used in the treatment of chronic myelogenous leukemia and malignant gastrointestinal stromal tumors, and has been explored in the treatment of thymic epithelial tumors. However, studies by Giaccon et al. [17] have shown that imatinib is not effective in thymic epit, helial tumor patients. In this study, imatinib was given to 7 patients (2 B3 type thymomas, 5 thymic carcinomas). Two patients were stable and five patients progressed with the median survival time as 4 months and the median survival time as 2 months. In another prospective clinical study, 11 patients with C-kit+ or PDGFR+ thymic carcinoma were enrolled and received imatinib [18]. While only three $\mathrm{SD}$ ( stable disease) patients $(27 \%)$ were found, and the median SD was 6 months. Hence, no effective cases were found in the study. However, arecent case report found that a 48-year-old patient with thymic carcinoma harboring $C$-kit gene mutationbenefited from imatinib therapy [19]. 


\subsection{Epithelial Growth Factor Receptor (EGFR)}

EGFR is a large transmembrane glycoprotein with a molecular weight of approximately $180 \mathrm{KDa}$ and ligandinduced tyrosine protein kinase activity. It is a member of a conserved receptor family of ErbB. A 2010 study [20] showed that EGFR mutations were rare in thymic epithelial tumors, leading to poor efficacy of EGFR targeting drug gefitinib in thymoma [21]. Li et al. [22] examined epidermal growth factor receptor (EGFR) and insulin-like growth factor-1 receptor (IGF-1R)in 63 thymomas and 15 thymic follicular hyperplasia cases using immunohistochemical EnVision two-step method and correlated with clinical significance. They found that high expression of EGFR and IGF-1R was related to the occurrence, development and invasion of thymoma, suggesting that the combined detection of EGFR and IGF-1R expression could provide useful information for assessing clinical stage and a reference for clinical treatment options. Christodoulou et al. reported a phase II clinical trial of erlotinib combined with bevacizumab therapy for 18 patients with thymoma or thymic carcinoma, who failed to respond to other treatments after recurrence. The study showed no effect was observed [23]. However, Takahashi et al. reported a 43-year-old female thymoma benefited from erlotinib therapy. The patient was treated with erlotinib when her disease recurred after six years after resection, and the tumor shrank significantly after 8 weeks of erlotinib treatment [24]. In other clinical studies, cetuximab therapy showedeffect inadvanced thymoma patients with EGFRprotein overexpression, but no EGFR gene mutation or amplification $[25,26]$. To date, there are few studies on anti-EGFR therapy for refractory thymoma, and the clinical outcome is still uncertain. Therefore, it may still worth exploring the expression and mutation of EGFR in thymoma in future.

\subsection{Insulin-like Growth Factor-1 Receptor (IGF-1R)}

IGF-1R is a transmembrane receptor involved in the regulation of cellular metabolism, growth and survival. A number of studies have shown that most thymic malignant tumors show moderate to high expression of IGF-1R. Omatsu et al. reported that IGF-1R expression was detected in $73 \%$ of patients with thymic carcinoma, while only $27 \%$ of patients with thymoma [27]. In another study with a cohort of 56 thymoma cases and 7 thymic carcinoma cases, moderate or strong IGF-1R expression was detected in $86 \%$ of thymic carcinoma patients, while only in $43 \%$ of thymoma patients [28]. Cixutumumab is a humanized antibody, which binds to
IGF-1R with high affinity and effectively mediates the internalization and degradation of IGF-1R receptor. It has been reported that cixutumumab can suppress tumor growth by blocking IGF-1R receptor function,thus inhibiting the activation of IGF-1R receptor and its signal transduction. At present, cixutumumab therapy for thymic tumor patients is still inearly stage. A recent study reported a phase I clinical study of cixutumumab treatment for a patient with metastaticthymoma and found the tumor sizehad a $10 \%$ reduction aftercixutumumab treatment with stable disease for one year [29]. Giaccone et al. reported a phase II clinical study of cixutumumab treatment in a cohort of 13 patients ( 5 cases of thymoma and 8 cases of thymic carcinoma), the results demonstrated that 8 patients (4 cases of thymoma, 4 cases of thymic adenocarcinoma) achieved stable disease, while 5 patients progressedafter cixutumumab therapy [30]. Rajan et al. reported a phase II clinical trial of cixutumumab therapy in a cohort of 49 patients with thymic epithelial tumors (37 cases of thymoma and 12 cases of thymic carcinoma). In the group of patients with thymoma, 5 cases had partial response, 28 cases had stable disease and 4 cases progressed. In the group of patients with thymic carcinoma, 5 cases had stable disease and 7 cases progressed, while no case had partial response [31].

\subsection{Histone Deacetylase (HDAC)}

HDAC plays an important role in modification of chromosome structures and regulation of gene expression. Belinostat, an DHAC inhibitor, inhibits cancer cell proliferation by increasing histone acetylation and p21 expression. Giaccone et al. reported a cohort of 14 thymomas and 8 thymic carcinomas treated with Belinostat therapy [30]. Among those, two thymoma patients achieved partial response, 13 patients were stable, and 6 patients progressed. Belinostat combined with chemotherapy is currently being investigated as first-line therapy for refractory advanced or recurrent thymomas.

\subsection{Vesicular Epithelial Growth Factor (VEGF)}

VEGF expression has been shown to be associated with tumor metastasis and staging [32]. Tomita et al. reported that VEGF proteins were detected in $56 \%$ $(11 / 18)$ of non-invasive thymomas, $55 \%(11 / 20)$ of invasive thymomas, and $62.8 \%$ ofthymic carcinomas. The study also revealed that there was a significant correlation between angiogenesis and invasiveness in patients with thymic epithelial tumors [33]. These 
results suggest a distinct correlation between VEGF expression and an increase of microvessel density.

\subsection{Other Markers}

In addition, somatostatin receptors, tropomyosin receptor kinase (Trk) and Cyclin-dependent kinase (CDK) inhibitors, steroid receptor coactivator (SRC) family and their ligands, exportin 1 (XPO1) inhibitor have been investigated as potential targets for treatment in thymic epithelial tumors [34-39]. It is difficult to enroll patients for clinical trials due to the low incidence and relative inertia of thymic epithelial tumors; therefore, these studies were restricted to individual case reports.

Remon $\mathrm{J}$ et al. found that all TET protein (teneleven translocation, TET) subtypes were CD22 positive, mainly in $A B$ type thymoma (68\%). They also found that $C D 26$ was expressed in type $A B$ thymoma $(68 \%)$ and type A thymoma (50\%), while CD52 was expressed more frequently in B2 and B3 thymoma. IGFR1 was the most common marker in thymic cancer samples (92\%), followed by EG5 (60\%). The expression of EG5 in thymic carcinoma was significantly higher than that in thymoma $(75 \%$ vs $38 \%$, $\mathrm{P}=0.026)$. These results suggest that agents targeting CD52, CD22, CD26 or EG5 [40] could be potentially effective in treating patients with thymic malignancies.

\section{MOLECULAR APPLICATIONS IN THE DIAGNOSIS OF THYMIC EPITHELIAL TUMORS}

In normal thymus, cathepsin V (CTV) and cathepsin $S$ (CTS) are expressed in cortical and medullary epithelial cells, respectively. Kiuchi $S$ et al. [41] investigated CTV and CTS expression using IHCs in 77 thymic epithelial tumors and found these two proteins were expressed differently in thymomas, with positive rates at $98 \%$ and $47 \%$, respectively. However, in thymic carcinomas, there was no significant difference in the expression of CTV and CTS, with positive rates at $41 \%$ and $47 \%$, respectively. CTS were expressed in type $A$ and type $A B$ thymoma, but not in type $B$ thymoma. Additionally, the expression of CTV and CTS in $A B$ type thymomas was significantly associated with histological features. The study also revealed that the recurrence rate of CTV-negative thymoma cases was higher than that of CTV-positive cases. The findings suggest that CTV and CTS can serve as auxiliary diagnostic and prognostic markers in thymic epithelial tumors.

Petrini et al. [42] analyzed 28 thymoma specimens using next-generation sequencing and identified the
GTF2I mutation (chromosome 7 c.74146970T>A) at a higher frequency (?\%) in type A thymoma compared to other type thymomas. Of the 274 thymoma specimens studied, GTF2I mutation was detected in $82 \%$ of type A and $74 \%$ of type $A B$ thymomas; however, this mutation rarely occurred in the aggressive subtype of thymomas.

Pax8 is a transcription factor involved in embryonic development of the thyroid, kidney, and mullerian systems and plays a role in tumorigenesis of these organs. Pax 8 protein is highly expressed in the primary and metastatic sites of thyroid, kidney and Mullerian tumors. Weissferdt A et al. [43] showed that Pax8 positive expression occurred in $77 \%$ of thymic carcinomas, $100 \%$ of type $A$ thymomas, and $93 \%$ of type B thymomas. They also found that Pax 8 was weakly expressed in epithelial cells of residual thymus tissue surrounding the tumor.

The potential use of squamous epithelial marker p40 and desmoglein core glycoprotein 3 (Desmoglein 3 , DSG-3) in diagnosing of thymic lesions is uncertain. Some studies have investigated their expression levels and patterns in 66 thymomas, 12 thymic squamous cell carcinomas, 6 undifferentiated thymic carcinomas, 5 proliferative thymuses and 5 normal thymuses, and found DSG-3 expression was useful in differentiating squamous cell carcinoma from thymoma. Of the 43 cases with both neoplastic and non-neoplastic thymus tissues in the same section, $26(60.5 \%)$ of the 43 cases where neoplastic and nonneoplastic thymus were present on the same slide, the presence/absence or distribution pattern of DSG-3 immunoreactivity was different in the 2 components, suggesting that this marker can be helpful in staging thymomas with incomplete encapsulation. The presence of DSG-3 positive and DSG-3 negative thymomas suggests these tumors may originate from two different types of thymic epithelial cells [44].

Another study found that the positive rates of $\beta 5 t$ were significantly different among B1 and B2, B3 types of thymomas. In $C D 20$-negative $A B$ thymoma, $\beta 5 t$ was mostly expressed in cytoplasm and perinuclear area, rarely in nucleoplasm. This unique expression pattern is useful in diagnosing $A B$ type thymoma. It has been shown that $\beta 5 t$ and CD20 were positive in bipolar type A thymomas, indicating their potential roles in identifying metaplastic thymoma [45].

Leisibach $\mathrm{P}$ et al. [46] found that cytoplasmic p21 expression was associated with overall survival and metastasis of malignant thymic epithelial tumors. 


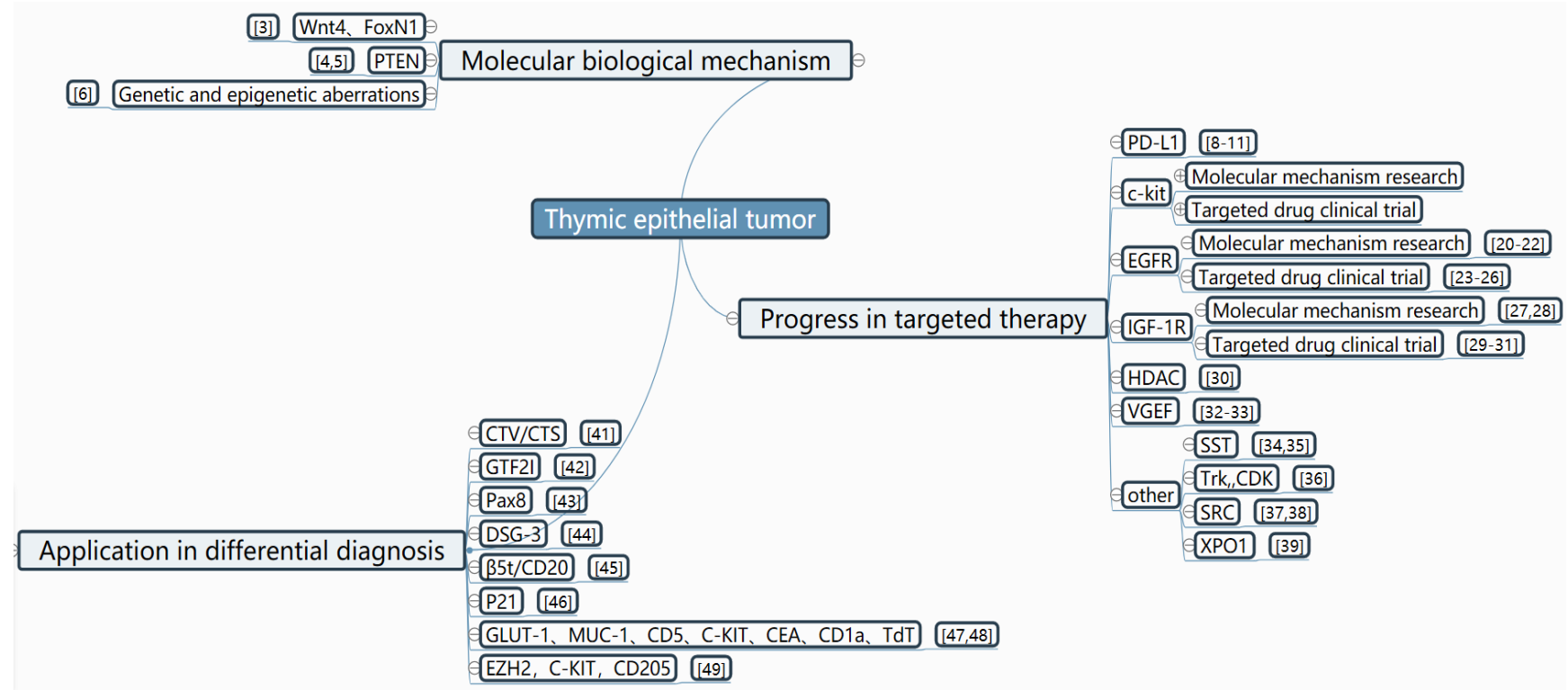

B3 thymoma and thymic squamous cell carcinoma have some overlapping histological features, making it difficult to differentiate these two entities from each other, especially in small biopsies. Mucin-1 (MUCIN-1, MUC-1) is a transmembrane mucin and is involved in tumor progression and outcome in a variety of malignancies. GLUT-1 is a member of the mammalian family of passive carriers of the Glucose transporter protein (GLUT) and functions as an energyindependent system for transporting glucose. These two proteins have been shown as useful markers for the diagnosis, progression and prognosis of various tumors. Su XY et al. [47] and Du J et al. [48] found there was a significant difference in the expression of GLUT-1, MUC-1, C-KIT, CD5, carcinoembryonic antigen (CEA), CD1a and Terminal Deoxynucleotidyl Transferase (TdT) between thymic squamous cell carcinoma and B3 thymoma. In thymic squamous cell carcinomas, GLUT-1 and MUC-1 were the most sensitive markers (100\%), followed by CK5 / 6 (95\%), C-KIT (90\%), P63 (85\%), CD5 (80\%) and CEA (75\%). Additionally, CD5, C-KIT and CEA were the most specific markers (100\%) for thymic squamous cell carcinomas, followed by MUC-1 (56.3\%), GLUT-1 (50\%), p63 (25\%) and CK5/6 (12.5\%). In B3 thymomas, the sensitivities were 100\%, 93.8\% and $87.5 \%$ for CK19, TdT and CD1a respectively, while the specificities were $100 \%$ for CD1a, $95 \%$ for TdT and $10 \%$ for CK19. Therefore, the combination of GLUT-1, MUC-1, CD5, C-KIT, CEA, CD1a and TdT can effectively differentiate thymic squamous cell carcinoma from B3 thymoma. Kim BS et al. [49] demonstrated that an immunohistochemical panel consisting of the cheese of zeste homolog 2 (EZH2), C-
KIT and CD205 was useful to differentiate thymic squamous cell carcinomas from type B3 thymomas. In a combined cohort analysis, the sensitivity and specificity of EZH2 were higher than those of C-KIT or CD205. The combined panel had the highest sensitivity and specificity at $96.3 \%$ and $100 \%$, which was significantly or marginally higher than those of EZH2, $\mathrm{C}-\mathrm{KIT}$, and CD205 alone, Indicating that EZH2 may be useful as a novel diagnostic marker for distinguishing thymic squamous cell carcinoma from thymomas.

Karube $\mathrm{Y}$ et al. reported folyl polyglutamate synthase (FPGS)/follicle-stimulating hormone (GGH) and vascular endothelial growth factor (VEGF) were elevated in thymic epithelial tumors and the expression levels were correlated with the degree of malignancy of B3 thymomas [50].

\section{SUMMARY}

Although multiple molecular markers and potential pathogenic pathways have recently been discovered in thymic epithelial tumors, targeted molecular therapy is still in its infancy. Future studies of signaling pathways and genetic mutations in thymic epithelial tumors are warranted to provide new targets for developing effective targeted therapies.

\section{SOURCE OF FUNDS}

Shandong Provincial Key Research and Development Program (2015GSF118168). Weifang Science and Technology Development Plan Project (2018YX012). 


\section{REFERENCES}

[1] Wu Jianguo, Cao Yong, Chen Bien,Wen Qiongna. 2013. Correlation between VEGF expression and PI3K/AKT pathway in Thymoma. Chinese And Foreign Medical Research, 11(17): 5-7.

[2] Travis William D, Brambilla Elisabeth, Burke Allen P, Marx Alexander, Nicholson Andrew G. 2015. Introduction to The 2015 World Health Organization Classification of Tumors of the Lung, Pleura, Thymus, and Heart. J Thorac Oncol, 10, 1240-1242.

https://doi.org/10.1097/JTO.0000000000000663

[3] Saito Motonobu, Fujiwara Yutaka, Asao Tetsuhiko, Honda Takayuki, Shimada Yoko, Kanai Yae, Tsuta Koji, Kono Koji, Watanabe Shunichi, Ohe Yuichiro, Kohno Takashi. 2017. The genomic and epigenomic landscape in thymic carcinoma. Carcinogenesis, 38, 1084-1091. https://doi.org/10.1093/carcin/bgx094

[4] Shi Feng. 2015.Expression of c-myc and PTEN genes in Thymoma[J]. Med Innov China, 12(32): 43-46.

[5] Masunaga Atsuko, Omatsu Mutsuko, Kunimura Toshiaki, Uematsu Shugo, Kamio Yoshito, Kitami Akihiko, Miyagi Yohei, Hiroshima Kenzo, Suzuki Takashi, 2017. PTEN Expression of and its pseudogene, and promoter methylation of in non-tumourous thymus and thymic tumours. J. Clin. Pathol, 70, 690-696.

https://doi.org/10.1136/jclinpath-2016-204220

[6] Wang Hai. 2016.Expression of Wnt4 and FoxN1 in Thymoma and its correlation with malignant degree of Thymoma. Tianjin Medical University.

[7] Meng Fanqing, Nie Ling. 2015. Interpretation of the 2014 IMTG thymic epithelial tumor classification consensus [J]. Chin J Clin Exp Pathol, 31(2): 121-123.

[8] Katsuya Yuki, Fujita Yu, Horinouchi Hidehito, Ohe Yuichiro, Watanabe Shun-Ichi, Tsuta Koji. 2015. Immunohistochemical status of PD-L1 in thymoma and thymic carcinoma. Lung Cancer, 88, 154-9. https://doi.org/10.1016/j.lungcan.2015.03.003

[9] Enkner Franz, Pichlhöfer Bettina, Zaharie Alexandru Teodor, Krunic Milica, Holper Tina Maria, Janik Stefan, Moser Bernhard, Schlangen Karin, Neudert Barbara, Walter Karin, Migschitz Brigitte, Müllauer Leonhard. 2017. Molecular Profiling of Thymoma and Thymic Carcinoma: Genetic Differences and Potential Novel Therapeutic Targets. Pathol. Oncol. Res, 23, 551-564. https://doi.org/10.1007/s12253-016-0144-8

[10] Tiseo Marcello, Damato Angela, Longo Lucia, Barbieri Fausto, Bertolini Federica, Stefani Alessandro, Migaldi Mario, Gnetti Letizia, Camisa Roberta, Bordi Paola, Buti Sebastiano, Rossi Giulio. 2017. Analysis of a panel of druggable gene mutations and of ALK and PD-L1 expression in a series of thymic epithelial tumors (TETs). Lung Cancer, 104, 24-30.

https://doi.org/10.1016/j.lungcan.2016.12.005

[11] Chen Yanmei, Zhang Yuping, Chai Xiaoling, Gao Jianfang, Chen Guorong, Zhang Weifen, Zhang Yunxiang. 2018. Correlation between the Expression of PD-L1 and Clinicopathological Features in Patients with Thymic Epithelial Tumors. Biomed Res Int, 2018, 5830547. https://doi.org/10.1155/2018/5830547

[12] Förster A, Grotha S P, Seeger J M, Rabenhorst A, Gehring M, Raap U, Létard S, Dubreuil P, Kashkar H, Walczak H, Roers A, Hartmann K. 2015. Activation of KIT modulates the function of tumor necrosis factor-related apoptosis-inducing ligand receptor (TRAIL-R) in mast cells. Allergy, 70, 764-74. https://doi.org/10.1111/all.12612

[13] Pan Chin-Chen, Chen Paul Chih-Hsueh, Chiang Hung. 2004. KIT (CD117) is frequently overexpressed in thymic carcinomas but is absent in thymomas. J. Pathol, 202, 375-81.

https://doi.org/10.1002/path.1514

[14] Kim Bo-Sung, Kim Jin Kuk, Kang Chang Hyun, Kim Young Tae, Jung Kyeong Cheon, Won Jae-Kyung. 2018. An immunohistochemical panel consisting of EZH2, C-KIT, and CD205 is useful for distinguishing thymic squamous cell carcinoma from type B3 thymoma. Pathol. Res. Pract, 214, 343-349.

https://doi.org/10.1016/j.prp.2018.01.009

[15] Aydiner Adnan, Toker Alper, Sen Fatma, Bicakci Ercan, Saglam Esra Kaytan, Erus Suat, Eralp Yesim, Tas Faruk, Oral Ethem Nezih, Topuz Erkan, Dilege Sukru. 2012. Association of clinical and pathological variables with survival in thymoma. Med. Oncol, 29, 2221-8. https://doi.org/10.1007/s12032-011-0101-z

[16] Schirosi L, Nannini N, Nicoli D, Cavazza A, Valli R, Buti S Garagnani L, Sartori G, Calabrese F, Marchetti A, Buttitta F, Felicioni L, Migaldi M, Rea F, Di Chiara F, Mengoli M C, Rossi G. 2012. Activating C-KIT mutations in a subset of thymic carcinoma and response to different c-KIT inhibitors. Ann. Oncol, 23, 2409-14.

\section{https://doi.org/10.1093/annonc/mdr626}

[17] Giaccone Giuseppe, Rajan Arun, Ruijter Rita, Smit Egbert, van Groeningen Cees, Hogendoorn Pancras C W. 2009. Imatinib mesylate in patients with $\mathrm{WHO}$ B3 thymomas and thymic carcinomas. J Thorac Oncol, 4, 1270-3. https://doi.org/10.1097/JTO.0b013e3181b6be57

[18] Palmieri Giovannella, Marino Mirella, Buonerba Carlo, Federico Piera, Conti Salvatore, Milella Michele, Petillo Luigi, Evoli Amelia, Lalle Maurizio, Ceribelli Anna, Merola Gerardina, Matano Elide, Sioletic Stefano, De Placido Sabino, Di Lorenzo Giuseppe, Damiano Vincenzo. 2012. Imatinib mesylate in thymic epithelial malignancies. Cancer Chemother. Pharmacol, 69, 309-15.

https://doi.org/10.1007/s00280-011-1690-0

[19] Buti Sebastiano, Donini Maddalena, Sergio Pietro, Garagnani Lorella, Schirosi Laura, Passalacqua Rodolfo, Rossi Giulio. 2011. Impressive response with imatinib in a heavily pretreated patient with metastatic C-KIT mutated thymic carcinoma. J. Clin. Oncol, 29, e803-5. https://doi.org/10.1200/JCO.2011.36.6427

[20] Girard Nicolas. 2010. Thymic tumors: relevant molecular data in the clinic. J Thorac Oncol, 5, S291-5. https://doi.org/10.1097/JTO.0b013e3181f209b9

[21] A. Kurup, M. Burns, S. Dropcho. 2005.Phase II study of gefitinib treatment in advanced thymic malignancies. J Clin Oncol, 23(16_suppl): 7068.

https://doi.org/10.1200/jco.2005.23.16 suppl.7068

[22] Li Wenshan, $\mathrm{Yu} \mathrm{Qi}, \mathrm{Li}$ Ning. 2016. Expression and significance of EGFR and IGF-1R in thymoma. Chin J Clin Exp Pathol,32(3): 297-300.

[23] Christodoulou C, Murray S, Dahabreh J, Petraki K, Nikolakopoulou A, Mavri A, Skarlos D. 2008. Response of malignant thymoma to erlotinib. Ann. Oncol, 19, 1361-2. https://doi.org/10.1093/annonc/mdn388

[24] Takahashi Eisuke, Koshiishi Haruya, Takahashi Masayoshi. 2011. [A response to erlotinib hydrochloride in the case of post-operative recurrent thymoma]. Gan To Kagaku Ryoho, 38, 2200-1.

[25] Farina Gabriella, Garassino Marina C, Gambacorta Marcello, La Verde Nicla, Gherardi Giorgio, Scanni Alberto. 2007. Response of thymoma to cetuximab. Lancet Oncol, 8, 44950. https://doi.org/10.1016/S1470-2045(07)70141-9

[26] Steele Nicola L, Plumb Jane A, Vidal Laura, Tjørnelund Jette, Knoblauch Poul, Rasmussen Annie, Ooi Chean Eng, BuhlJensen Peter, Brown Robert, Evans T R Jeffry, DeBono 
Johann S. 2008. A phase 1 pharmacokinetic and pharmacodynamic study of the histone deacetylase inhibitor belinostat in patients with advanced solid tumors. Clin. Cancer Res, 14, 804-10.

https://doi.org/10.1158/1078-0432.CCR-07-1786

[27] Omatsu Mutsuko, Kunimura Toshiaki, Mikogami Tetsuya, Hamatani Shigeharu, Shiokawa Akira, Masunaga Atsuko, Kitami Akihiko, Suzuki Takashi, Kadokura Mitsutaka, Morohoshi Toshio. 2012. Immunohistochemical analysis of thymic carcinoma focusing on the possibility of molecular targeted and hormonal therapies. Gen Thorac Cardiovasc Surg, 60, 803-10.

https://doi.org/10.1007/s11748-012-0160-x

[28] Girard Nicolas, Teruya-Feldstein Julie, Payabyab Eden C, Riely Gregory J, Rusch Valerie W, Kris Mark G, Zakowski Maureen F. 2010. Insulin-like growth factor-1 receptor expression in thymic malignancies. J Thorac Oncol, 5, 143946. https://doi.org/10.1097/JTO.0b013e3181e392a8

[29] Haluska Paul, Shaw Heather M, Batzel Gretchen N, Yin Donghua, Molina Julian R, Molife L Rhoda, Yap Timothy A, Roberts M Luisa, Sharma Amarnath, Gualberto Antonio, Adjei Alex A, de Bono Johann S. 2007. Phase I dose escalation study of the anti insulin-like growth factor-I receptor monoclonal antibody CP-751,871 in patients with refractory solid tumors. Clin. Cancer Res, 13, 5834-40. https://doi.org/10.1158/1078-0432.CCR-07-1118

[30] Giaccone Giuseppe, Rajan Arun, Berman Arlene, Kelly Ronan J, Szabo Eva, Lopez-Chavez Ariel, Trepel Jane, Lee Min-Jung, Cao Liang, Espinoza-Delgado Igor, Spittler John, Loehrer Patrick J. 2011. Phase II study of belinostat in patients with recurrent or refractory advanced thymic epithelial tumors. J. Clin. Oncol, 29, 2052-9. https://doi.org/10.1200/JCO.2010.32.4467

[31] A. Rajan, Arlene Berman, R. J. Kelly, Ariel Lopez-Chavez. 2010. Phase II study of the insulin-like growthfactor-1 receptor (IGF-1R) antibody cixutumumab (C) in patients (pts) with thymoma (T) and thymic carcinoma (TC) . J Clin Oncol, 28S: Abstract e17525.

https://doi.org/10.1200/jco.2010.28.15 suppl.e17525

[32] Cimpean Anca Maria, Raica Marius, Encica Svetlana, Cornea Remus, Bocan Viorica. 2008. Immunohistochemical expression of vascular endothelial growth factor A (VEGF), and its receptors (VEGFR1, 2) in normal and pathologic conditions of the human thymus. Ann. Anat, 190, 238-45. https://doi.org/10.1016/j.aanat.2007.05.003

[33] Tomita Masaki, Matsuzaki Yasunori, Edagawa Masao, Maeda Masayuki, Shimizu Tetsuya, Hara Masaki, Onitsuka Toshio. 2002. Correlation between tumor angiogenesis and invasiveness in thymic epithelial tumors. J. Thorac. Cardiovasc. Surg, 124, 493-8. https://doi.org/10.1067/mtc.2002.124389

[34] Loehrer Patrick J, Wang Wei, Johnson David H, Aisner Seena C, Ettinger David S, Eastern Cooperative Oncology Group Phase II Trial. 2004. Octreotide alone or with prednisone in patients with advanced thymoma and thymic carcinoma: an Eastern Cooperative Oncology Group Phase II Trial. J. Clin. Oncol, 22, 293-9. https://doi.org/10.1200/JCO.2004.02.047

[35] Palmieri Giovannella, Buonerba Carlo, Federico Piera, Formisano Luigi, Nappi Lucia, Di Lorenzo Giuseppe, Marino Mirella, Damiano Vincenzo. 2012. Everolimus plus longacting somatostatin analogs in thymic epithelial malignancies. World J Clin Oncol, 3, 111-5. https://doi.org/10.5306/wjco.v3.i7.111

[36] Gbolahan Olumide B, Porter Ryan F, Salter John T, Yiannoutsos Constantin, Burns Matthew, Chiorean E Gabriella, Loehrer Patrick J. 2018. A Phase II Study of Pemetrexed in Patients with Recurrent Thymoma and Thymic Carcinoma. J Thorac Oncol, 13, 1940-1948. https://doi.org/10.1016/j.jtho.2018.07.094
[37] Gubens Matthew A, Burns Matthew, Perkins Susan M, Pedro-Salcedo Melanie San, Althouse Sandy K, Loehrer Patrick J, Wakelee Heather A. 2015. A phase II study of saracatinib (AZD0530), a Src inhibitor, administered orally daily to patients with advanced thymic malignancies. Lung Cancer, 89, 57-60.

https://doi.org/10.1016/j.lungcan.2015.04.008

[38] Chuah Charles, Lim Tse Hui, Lim Alvin Soon Tiong, Tien Sim Leng, Lim Chong Hee, Soong Richie, Lee Francis, Linn Yeh Ching, Goh Yeow Tee, Cheah Foong Koon, Loh Alwin Hwai Liang. 2006. Dasatinib induces a response in malignant thymoma. J. Clin. Oncol, 24, e56-8. https://doi.org/10.1200/JCO.2006.08.8963

[39] Conforti Fabio, Wang Yisong, Rodriguez Jose A, Alberobello Anna Teresa, Zhang Yu-Wen, Giaccone Giuseppe. 2015. Molecular Pathways: Anticancer Activity by Inhibition of Nucleocytoplasmic Shuttling. Clin. Cancer Res, 21, 4508-13. https://doi.org/10.1158/1078-0432.CCR-15-0408

[40] Remon J, Abedallaa N, Taranchon-Clermont E, Bluthgen V, Lindsay C R, Besse B, Thomas de Montpréville V. 2017. CD52, CD22, CD26, EG5 and IGF-1R expression in thymic malignancies. Lung Cancer, 108, 168-172.

https://doi.org/10.1016/j.lungcan.2017.03.019

[41] Kiuchi Shizuka, Tomaru Utano, Ishizu Akihiro, Imagawa Makoto, Kiuchi Takayuki, Iwasaki Sari, Suzuki Akira, Otsuka Noriyuki, Deguchi Takahiro, Shimizu Tomohiro, Marukawa Katsuji, Matsuno Yoshihiro, Kasahara Masanori. 2017. Expression of cathepsins $\mathrm{V}$ and $\mathrm{S}$ in thymic epithelial tumors. Hum. Pathol, 60, 66-74. https://doi.org/10.1016/j.humpath.2016.09.027

[42] Petrini lacopo, Meltzer Paul S, Kim In-Kyu, Lucchi Marco, Park Kang-Seo, Fontanini Gabriella, Gao James, Zucali Paolo A, Calabrese Fiorella, Favaretto Adolfo, Rea Federico, Rodriguez-Canales Jaime, Walker Robert L, Pineda Marbin, Zhu Yuelin J, Lau Christopher, Killian Keith J, Bilke Sven, Voeller Donna, Dakshanamurthy Sivanesan, Wang Yisong, Giaccone Giuseppe. 2014. A specific missense mutation in GTF2I occurs at high frequency in thymic epithelial tumors. Nat. Genet, 46, 844-9.

https://doi.org/10.1038/ng.3016

[43] Weissferdt Annikka, Moran Cesar A. 2011. Pax8 expression in thymic epithelial neoplasms: an immunohistochemical analysis. Am. J. Surg. Pathol, 35, 1305-10. https://doi.org/10.1097/PAS.0b013e3182260735

[44] Walts Ann E, Hiroshima Kenzo, Marchevsky Alberto M. 2015. Desmoglein 3 and p40 immunoreactivity in neoplastic and nonneoplastic thymus: a potential adjunct to help resolve selected diagnostic and staging problems. Ann Diagn Pathol, 19, 216-20. https://doi.org/10.1016/j.anndiagpath.2015.04.006

[45] Li Songmei, Tan Wei, Huang Shubin, Hu Xiangyang. 2016. Expression and significance of $\beta 5 t, p 63$ and CD20 in Thymoma . Chin J Clin Exp Pathol, 32(3):292-296.

[46] Leisibach Priska, Schneiter Didier, Soltermann Alex, Yamada Yoshi, Weder Walter, Jungraithmayr Wolfgang. 2016. Prognostic value of immunohistochemical markers in malignant thymic epithelial tumors. J Thorac Dis, 8, 25802591.

https://doi.org/10.21037/jtd.2016.08.82

[47] Su Xue-Ying, Wang Wei-Ya, Li Jin-Nan, Liao Dian-Ying, Wu Wei-Lu, Li Gan-Di. 2015. Immunohistochemical differentiation between type B3 thymomas and thymic squamous cell carcinomas. Int J Clin Exp Pathol, 8, 5354-62.

[48] Du Ms Jun, Shen Qin, Yin Honglin, Rao Qiu, Zhou Mr Xiaojun. 2016. Diagnostic roles of MUC1 and GLUT1 in differentiating thymic carcinoma from type B3 thymoma. Pathol. Res. Pract, 212, 1048-1051. https://doi.org/10.1016/j.prp.2016.09.005 
[49] Kim Bo-Sung, Kim Jin Kuk, Kang Chang Hyun, Kim Young Tae, Jung Kyeong Cheon, Won Jae-Kyung. 2018. An immunohistochemical panel consisting of EZH2, C-KIT, and CD205 is useful for distinguishing thymic squamous cell carcinoma from type B3 thymoma. Pathol. Res. Pract, 214, 343-349.

https://doi.org/10.1016/j.prp.2018.01.009
[50] Karube Yoko, Kobayashi Satoru, Maeda Sumiko, Sado Tetsu, Ishihama Hiromi, Chida Masayuki. 2016. Tumorrelated gene expression levels in thymic carcinoma and Type B3 thymoma. J Cardiothorac Surg, 11, 85.

https://doi.org/10.1186/s13019-016-0468-1

Received on 12-11-2019

Accepted on 11-12-2019

Published on 19-12-2019

DOI: http://dx.doi.org/10.30683/1929-2279.2019.08.02

(C) 2019 Ruiz et al.; Licensee Neoplasia Research.

This is an open access article licensed under the terms of the Creative Commons Attribution Non-Commercial License (http://creativecommons.org/licenses/by-nc/3.0/) which permits unrestricted, non-commercial use, distribution and reproduction in any medium, provided the work is properly cited. 